\title{
A Study on the Correlation between Stressors and Psychological Behaviors of Nurses in Support of COVID-19 Ward
}

\author{
Yongyan Ding1, Mo Fu², Juan Ding2 \\ ${ }^{1}$ Department of Gynecology, Jingzhou Central Hospital, Jingzhou, China \\ ${ }^{2}$ Nursing Department, Jingzhou Central Hospital, Jingzhou, China \\ Email: 1320037305@qq.com
}

How to cite this paper: Ding, Y.Y., Fu, M. and Ding, J. (2021) A Study on the Correlation between Stressors and Psychological Behaviors of Nurses in Support of COVID-19 Ward. Yangtze Medicine, 5, 194-206.

https://doi.org/10.4236/ym.2021.53019

Received: January 25, 2021

Accepted: September 14, 2021

Published: September 17, 2021

Copyright $\odot 2021$ by author(s) and Scientific Research Publishing Inc. This work is licensed under the Creative Commons Attribution International License (CC BY 4.0).

http://creativecommons.org/licenses/by/4.0/

\begin{abstract}
COVID-19 has been a hot topic of research in medical journals. There are many articles which have been published on the genetic sequencing, pathology and other medical conditions associated with COVID-19, however, there are very few articles which acknowledge the psychological and behavioral aspects of front-line medical workers especially nurses. The purpose of the study is to understand the stressors, psychological behavior status and the correlation between psychological behavior and stressors for nurses who served in COVID-19 ward. The general information questionnaire, the nurse job stressor scale in Chinese, and the emergency public health incident questionnaire were used to survey 282 nurses in Jingzhou Central Hospital who supported COVID-19 ward. The result shows that the average work stress of nurses in this study was $1.40 \pm 0.54$ points. The top 3 dimensions were conceptual stress $(1.67 \pm 0.68)$, working environment stress $(1.57 \pm 0.60)$, and occupational hazard stress $(1.57 \pm 0.66)$. With different marital status, the average scores of stress are different, and the difference is statistically significant $(t=2.139, P=0.034)$. For nurses in COVID-19 ward who faced sudden public health crisis, the self-evaluated psychological score of nurses in COVID-19 ward averages 1.40 with a deviation of 0.54 . In our study, fear had the highest influence on psychological behavior, while the lowest was the compulsive-anxiety factor. There was a positive correlation between the stressor scores and the psychological behavior scores $(\mathrm{P}<0.01)$. The conclusion is that the pressure of nurses supporting COVID-19 ward is under moderate pressure, and the psychological behavior is moderately responded with a few nurses having psychological behavior deviation.
\end{abstract}

\section{Keywords}

COVID-19, Nurses, Psychological Behavior, Stressor, Correlation 


\section{Introduction}

Work stress is the physical and mental stress of practitioners caused by the imbalance between professional requirements and individual endurance [1]. Since the outbreak of COVID-19, the majority of medical staff have been fighting at the forefront of epidemic prevention and control. Due to the high contagiousness of COVID-19 and the limitation of understanding of the disease [2], it has a huge impact not only on the body but also the mind of medical staff. It has also attracted great attention from government departments as well as the public. Our hospital is an excellent prefectural-level comprehensive hospital with 1792 open beds, 1353 nursing staff, and 52 clinical departments. From the admission of the first patient with COVID-19 on January 16, 2020, the hospital has transformed 7 general departments for the admission of patients with COVID-19. A total of 136 confirmed patients were admitted, including 38 critically ill patients, and 32 suspected patients. The Nursing Department has successively assigned 350 nurses from internal medicine, surgery, obstetrics and gynecology, oncology, infectious diseases, and intensive care units to support the nursing work of the COVID-19 ward. In the face of this sudden public health crisis, we need to understand the stressors of nurses, their psychological and emotional status, as well as individuals whose psychological behavior has altered. We also need to conduct psychological intervention in time with the understanding of their psychological behavior and stress, the relevance to the stressor, and take corresponding countermeasures to avoid the seriousness and sustainable development of psychological crisis [3], in order to effectively protect the physical and mental health of medical staff.

\section{Objects and Methods}

\subsection{Research Subjects}

In February 2020, the convenience sampling method was used to survey the nurses who supported the covid-19 ward in a tertiary excellent general hospital in Jingzhou City, Hubei Province. The nursing staff who agreed to participate in this study were included.

Inclusion criteria: 1) COVID-19 patients who are being treated; 2) Volunteer who participated in study; 3) Ignorance and communication barrier. Exclusion criteria: Those nurses who worked less than 1 week in the COVID-19 ward.

\subsection{Survey Tools}

The questionnaire includes three parts: the general information questionnaire, the Chinese version of the nurses' job stressor scale, and the emergency public health incident questionnaire. More details are described as follows.

\subsubsection{General Information Questionnaire}

Self-designed, including the gender, age, education, marital status, working years of the surveyor, department before working in COVID-19 ward, etc. 


\subsubsection{The Chinese Version of the Nurses' Work Stressor Scale}

The "Chinese Nurses' Work Stressor Scale" was adopted [4] [5]. Based on the research of the scale, consulting experts and referring to relevant domestic and foreign literature [6]-[11], a supplementary pressure source scale was designed and generated by us. A total of 39 items were divided into seven dimensions: professional, psychological, working environment, interpersonal relationship, conceptual pressure, occupational hazards, and family aspects. The score ranges from 0 to 3, where 0 represents no stress, 1 represents mild stress, 2 represents moderate stress, and 3 represents severe stress. The total score of each dimension divided by the number of items is the score of that dimension. The theoretical maximum value is 3 and the theoretical minimum value is 0 . The higher the score, the greater the degree of stress. The total Cronbach's $\alpha$ coefficient of this scale is 0.85 .

\subsubsection{Chinese Version of the Questionnaire on Public Health Emergencies [12] [13]}

This questionnaire was compiled during the atypical pneumonia (SARS) epidemic in 2003. It is used to evaluate the psychological behavior after public health emergency. According to actual needs, it can be used for individual or group survey. There are 27 items which is divided into 5 dimensions: Depression, Neurasthenia, Fear, Compulsive-anxiety, and hypochondriasis. According to the degree of psychological behavior (no, mild, moderate, severe) and frequency (occasionally, sometimes, often, always), the corresponding score ranges from 0 to 3 . The total score of each dimension divided by the number of items is the score of the dimension. The theoretical maximum value is 3 , and the theoretical minimum value is 0 . The retest correlation coefficient of the five dimensions is between $0.401-0.920$, and the total score is retested correlation coefficient is 0.631 . The Cronbach's $\alpha$ coefficient of the entire questionnaire is 0.692 . The Cronbach's $\alpha$ coefficient of the five dimensions is between $0.755-0.804$.

\subsection{Survey Method}

A questionnaire survey was conducted, a preliminary survey was organized in the early stage to improve the questionnaire design. All head nurses in the covid-19 ward were systematically trained and instructed. The questionnaire survey was distributed uniformly in the We-Chat group of the COVID-19 ward, and the survey was completed with the guide of head Nurses. 282 out of 350 nursing staff in the COVID-19 ward area agreed to participate in the survey and 282 questionnaires were recovered. The response rate was $100 \%$, and the effective response rate was $100 \%$.

\subsection{Statistical Methods}

The original data of the questionnaire was exported in EXECL format, and SPSS 18.0 was used for data processing. The measurement data is expressed by $x \pm S$, and the counting data is described by frequency and composition ratio. Inde- 
pendent sample t test, single factor ANOVA, Pearson correlation analysis and other analysis methods have been applied in the analysis.

\section{Results}

\subsection{General Information}

The general information of the participants (Nurses worked in covid-19 wards) are: Age $30.58+5.03$ years, working experience of $8.63+5.69$ years; Educational background: 7 Junior college students (2.48\%), 271 undergraduate students (96.10\%), 4 postgraduate students (1.42\%); Professional title: 8 vice senior and above nurses (2.84\%), 90 nurse incharge (31.91\%), 146 primary nurse (51.77\%), 38 nurses (13.48\%); Department of Nurses in which they belonged to previously are 62 from ICU (21.99\%), 17 from infectious disease department (6.03\%), 16 from obstetrics and gynecology department (5.67\%), 42 from oncology department (14.89\%) and 74 from general surgery department (5.67\%), 71 from general internal medicine department (25.18\%); Marital status: 99 of them were unmarried (35.3\%), 183 were married (64.89\%). See Table 1 for details.

Table 1. Baseline data of nurses supporting COVID-19 area $(\bar{x} \pm \mathrm{S}, \mathrm{n}, \%)$.

\begin{tabular}{|c|c|}
\hline Groups & Statistics \\
\hline Age $(\bar{x} \pm S)$ & $30.58 \pm 5.03$ \\
\hline Years of service $(\bar{x} \pm S)$ & $8.63 \pm 5.69$ \\
\hline \multicolumn{2}{|l|}{ Professionaltitle (n, \%) } \\
\hline Vice senior and above & $8(2.84)$ \\
\hline nurse-in-charge & $90(31.91)$ \\
\hline primary nurse & $146(51.77)$ \\
\hline Nurse & $38(13.48)$ \\
\hline \multicolumn{2}{|l|}{ Education (n, \%) } \\
\hline Junior college & $7(2.48)$ \\
\hline Undergraduate & $271(96.10)$ \\
\hline Post-Graduate & $4(1.42)$ \\
\hline \multicolumn{2}{|l|}{ Former Departments (n, \%) } \\
\hline ICU & $62(21.99)$ \\
\hline Infectious Diseases & $17(6.03)$ \\
\hline Obstetrics and Gynecology & $16(5.67)$ \\
\hline Oncology & $42(14.89)$ \\
\hline General Surgery & $74(26.24)$ \\
\hline General Internal Medicine & $71(25.18)$ \\
\hline \multicolumn{2}{|l|}{ Marital status (n, \%) } \\
\hline Yes & $183(64.89)$ \\
\hline No & $99(35.11)$ \\
\hline
\end{tabular}




\subsection{Stressors for Nurses Working in COVID-19 Ward}

In this study, the average score of stress was $40 \pm 0.54$. The dimension with the highest score on the stressor scale was conceptual stress, and the lowest dimension was family stress (Table 2); the top 10 with highest score in the nurse stressor scale are listed in Table 3 , and the average score is $>1.50$.

\subsection{Correlation between Stressors, Psychological Behaviors and General Information}

Single factor ANOVA or T test were used to analyze the mean score of age, working years, professional title, educational background, Department, Marital status and the average score of total stressors and nurses' psychological behavior. It was found that the average score of stressors of nurses with different marital status was different and the difference was statistically significant $(t=2.139, \mathrm{Pt}=$ 0.034). In addition, the psychological and behavioral responses of nurses with different marital status were statistically significant $(t=2.004, P=0.047)$. See Table 4 for details.

Table 2. Distribution and ranking of all dimensions of stressors for nurses $(\mathrm{n}=282, \bar{X}$ $\pm \mathrm{S})$.

\begin{tabular}{ccc}
\hline Dimension & Average Score & Rank \\
\hline Conceptual stress & $1.67 \pm 0.68$ & 1 \\
Occupational hazards & $1.57 \pm 0.66$ & 2 \\
Work environment & $1.57 \pm 0.60$ & 3 \\
Psychological aspects & $1.39 \pm 0.67$ & 4 \\
Professional & $1.36 \pm 0.61$ & 5 \\
Interpersonal relationships & $0.95 \pm 0.59$ & 6 \\
Family aspects & $0.78 \pm 0.67$ & 7 \\
\hline
\end{tabular}

Table 3. Average and ranking of top ten stress sources for nurses $(\mathrm{n}=282, \bar{x} \pm S)$.

\begin{tabular}{|c|c|c|c|}
\hline Entry & Dimensions & $\begin{array}{l}\text { Divide } \\
\text { equally }\end{array}$ & Sort \\
\hline Worried about family & Conceptual stress & $1.87 \pm 0.89$ & 1 \\
\hline Worried about being infected & Conceptual stress & $1.83 \pm 0.85$ & 2 \\
\hline I want to go back but dare not/can't go home & Conceptual stress & $1.80 \pm 0.95$ & 3 \\
\hline $\begin{array}{c}\text { High-risk operations } \\
\text { (Nebulization, oral care, tracheal intubation, etc.) }\end{array}$ & occupational hazard & $1.77 \pm 0.87$ & 4 \\
\hline $\begin{array}{l}\text { Irregular working hours (such as unable to eat, } \\
\text { drink, go to the toilet on time, etc.) }\end{array}$ & working condition & $1.77 \pm 0.74$ & 5 \\
\hline Discomfort of protective clothing and supplies & working condition & $1.76 \pm 0.74$ & 6 \\
\hline Facing the death of a patient & Conceptual stress & $1.75 \pm 0.87$ & 7 \\
\hline $\begin{array}{l}\text { Inconvenient protective gloves and other supplies, } \\
\text { and potential needle stick injuries }\end{array}$ & occupational hazard & $1.73 \pm 0.80$ & 8 \\
\hline Separation from family & Conceptual stress & $1.67 \pm 0.92$ & 9 \\
\hline Worry about mistakes at work & Professional & $1.60 \pm 0.78$ & 10 \\
\hline
\end{tabular}


Table 4. Comparison of general data on the stressors and psychological behaviors of nurses in the COVID-19 ward.

\begin{tabular}{|c|c|c|c|c|c|c|c|}
\hline \multirow{2}{*}{ Dimension } & \multirow{2}{*}{$\begin{array}{l}\text { Number } \\
\text { of people }\end{array}$} & \multicolumn{3}{|c|}{$\begin{array}{l}\text { Nurse Work } \\
\text { Stress Source }\end{array}$} & \multicolumn{3}{|c|}{$\begin{array}{c}\text { Psychological } \\
\text { behavior of nurses }\end{array}$} \\
\hline & & $\begin{array}{l}\text { Divide } \\
\text { equally }\end{array}$ & $\mathrm{F} / \mathrm{t}$ & $\begin{array}{c}\mathrm{P} \\
\text { value }\end{array}$ & $\begin{array}{l}\text { Average } \\
\text { Score }\end{array}$ & $\mathrm{F} / \mathrm{t}$ & $\begin{array}{c}\mathrm{P} \\
\text { value }\end{array}$ \\
\hline \multicolumn{8}{|l|}{ Age } \\
\hline $20-30$ & 158 & $1.37 \pm 0.51$ & \multirow{3}{*}{0.287} & \multirow{3}{*}{0.751} & $0.87 \pm 0.51$ & \multirow{3}{*}{0.531} & \multirow{3}{*}{0.0 .589} \\
\hline $31-40$ & 115 & $1.42 \pm 0.55$ & & & $0.97 \pm 0.58$ & & \\
\hline$\geq 41$ & 9 & $1.54 \pm 1.15$ & & & $1.00 \pm 1.17$ & & \\
\hline \multicolumn{8}{|l|}{ Years of service } \\
\hline $1-5$ & 98 & $1.26 \pm 0.47$ & \multirow{4}{*}{1.607} & \multirow{4}{*}{0.191} & $0.79 \pm 0.53$ & \multirow{4}{*}{1.486} & \multirow{4}{*}{0.221} \\
\hline $6-10$ & 100 & $1.47 \pm 0.57$ & & & $0.97 \pm 0.54$ & & \\
\hline $11-15$ & 62 & $1.49 \pm 0.51$ & & & $1.04 \pm 0.57$ & & \\
\hline$\geq 16$ & 21 & $1.33 \pm 0.75$ & & & $0.86 \pm 0.72$ & & \\
\hline \multicolumn{8}{|l|}{ Professional title } \\
\hline Vice nurses and above & 8 & $1.49 \pm 1.00$ & \multirow{4}{*}{1.301} & \multirow{4}{*}{0.277} & $0.94 \pm 1.02$ & \multirow{4}{*}{0.433} & \multirow{4}{*}{0.730} \\
\hline nurse-in-charge & 90 & $1.50 \pm 0.52$ & & & $0.98 \pm 0.54$ & & \\
\hline primary nurse & 146 & $1.36 \pm 0.53$ & & & $0.87 \pm 0.54$ & & \\
\hline nurse & 38 & $1.22 \pm 0.49$ & & & $0.85 \pm 0.57$ & & \\
\hline \multicolumn{8}{|l|}{ Education } \\
\hline junior college & 7 & $1.47 \pm 0.49$ & \multirow{3}{*}{0.419} & \multirow{3}{*}{0.659} & $0.93 \pm 0.46$ & \multirow{3}{*}{0.003} & \multirow{3}{*}{0.997} \\
\hline Nndergraduate & 271 & $1.40 \pm 0.55$ & & & $0.91 \pm 0.57$ & & \\
\hline Post-graduate & 4 & $1.36 \pm 0.42$ & & & $0.90 \pm 0.42$ & & \\
\hline \multicolumn{8}{|l|}{ Former-departments } \\
\hline ICU & 62 & $1.44 \pm 0.54$ & \multirow{6}{*}{0.793} & \multirow{6}{*}{0.557} & $0.90 \pm 0.58$ & \multirow{6}{*}{0.661} & \multirow{6}{*}{0.654} \\
\hline Infectious Diseases & 17 & $1.48 \pm 0.66$ & & & $0.92 \pm 0.46$ & & \\
\hline Obstetrics and Gynecology & 16 & $1.73 \pm 1.01$ & & & $1.21 \pm 1.04$ & & \\
\hline Oncology & 42 & $1.34 \pm 0.49$ & & & $0.98 \pm 0.65$ & & \\
\hline General Surgery & 74 & $1.32 \pm 0.48$ & & & $0.82 \pm 0.38$ & & \\
\hline General Internal Medicine & 71 & $1.36 \pm 0.49$ & & & $0.91 \pm 0.56$ & & \\
\hline \multicolumn{8}{|l|}{ Marital status } \\
\hline Yes & 183 & $1.47 \pm 0.54$ & & & $0.98 \pm 0.56$ & & \\
\hline No & 99 & $1.26 \pm 0.53$ & 2.109 & 0.004 & $0.78 \pm 0.53$ & 2.004 & 0.047 \\
\hline
\end{tabular}

\subsection{Psychological Behavior of Nurses in the COVID-19 Ward in Response to Sudden Public Health Emergency}

After sudden public health emergency, the average score of psychological behavior of nurses was $(1.40 \pm 0.54)$, the highest dimension of psychological behavior was fear factor, and the lowest dimension was compulsive-anxiety factor. See Table 5 . 
Table 5. Psychological behavior of nurses after sudden public health emergency $(\mathrm{n}=282$, $\bar{x} \pm \mathrm{S})$.

\begin{tabular}{ccc}
\hline Dimension & Average Score & Rank \\
\hline Fear & $1.46 \pm 0.68$ & 1 \\
Depression & $1.02 \pm 0.78$ & 2 \\
Neurasthenia & $0.91 \pm 0.70$ & 3 \\
Hypochondria & $0.86 \pm 0.73$ & 4 \\
Compulsive-anxiety & $0.70 \pm 0.63$ & 5 \\
\hline
\end{tabular}

\subsection{Correlation Analysis of Stress Sources and Psychological Behavior}

A positive correlation was found between stress source score and psychological behavior score $(\mathrm{P}<0.01)$, that is, the higher the psychological behavior scores, the higher the stressor scores. See Table 6.

\section{Discussion}

\subsection{Self-Assessment of the Stressors of Nurses in the COVID-19 Ward}

In this study, the stress of nurses was $1.40 \pm 0.54$ points and the stress of nurses working in the COVID-19 area was moderate. The top 3 dimensions were conceptual stress (1.67 \pm 0.68$)$, occupational hazard stress (1.57 \pm 0.66$)$, and working environment stress $(1.57 \pm 0.60)$. The top 10 entities are: worry about family members (1.87 \pm 0.89$)$; Fear of being infected (1.83 \pm 0.85$)$; Want to go back and Dare not/can't to go home (1.80 \pm 0.95$)$; High risk operations (atomization, oral care, tracheal intubation, etc.), Increased risk of infection (1.77 \pm 0.87$)$; Irregular working hours (e.g. failure to eat, drink, go to the toilet, etc.) (1.77 \pm 0.74$)$; Discomfort with protective clothing and supplies (1.76 \pm 0.74$)$; In the face of death $(1.75 \pm 0.87)$; inconvenient protective gloves and other equipment and potential needle stab wounds $(1.73 \pm 0.80)$. Details of the stressors and possible solutions are discussed as follows.

\subsubsection{Worry about the Family}

Ranked first among nurses' stressors, it belongs to the dimension of conceptual stress. It is related to the characteristics of COVID-19, which is a new type of corona virus infection, which is generally susceptible to infection in the population and easy to transmit at home. The professional mission of nurses is to save the dead and heal the wounded. In the face of this epidemic, professional ethics urged nurses to devote themselves to the fight against the epidemic without hesitation, but at the same time they were worried that they would be infected and may transmit the infection to the entire family, causing great pressure on nurses [14] [15]. This required the hospital leaders and nursing managers to: Support the nurses in the COVID-19 ward, by arranging a single room in a hotel so they can be isolated from their families to ensure their safety during their working 
Table 6. Relationship between stressors and nurses' psychological behavior ( $\gamma$ value).

\begin{tabular}{|c|c|c|c|c|c|c|}
\hline Dimension & Depression & Neurasthenia & Fear & Compulsive-anxiety & Hypochondria & Psychological behavior \\
\hline Professional & $0.546^{*}$ & $0.537^{*}$ & $0.621^{*}$ & $0.604^{*}$ & $0.512^{*}$ & $0.639^{*}$ \\
\hline Psychological aspects & $0.469^{*}$ & $0.544^{*}$ & $0.600^{*}$ & $0.518^{*}$ & $0.508^{\star}$ & $0.592^{*}$ \\
\hline Work environment & $0.561^{\star}$ & $0.580^{*}$ & $0.674^{*}$ & $0.604^{*}$ & $0.540^{*}$ & $0.670^{*}$ \\
\hline Interpersonal relationships & $0.393^{*}$ & $0.466^{*}$ & $0.441^{*}$ & $0.525^{*}$ & $0.452^{\star}$ & $0.503^{*}$ \\
\hline Conceptual stress & $0.499^{*}$ & $0.614^{*}$ & $0.715^{*}$ & $0.546^{*}$ & $0.515^{*}$ & $0.656^{*}$ \\
\hline Occupational hazards & $0.585^{*}$ & $0.585^{*}$ & $0.676^{*}$ & $0.596^{*}$ & $0.532^{*}$ & $0.677^{*}$ \\
\hline Family aspects & $0.527^{*}$ & $0.453^{*}$ & $0.426^{*}$ & $0.560^{*}$ & $0.397^{*}$ & $0.540^{*}$ \\
\hline Total pressure source & $0.613^{*}$ & $0.650^{*}$ & $0.726^{*}$ & $0.670^{\star}$ & $0.589^{\star}$ & $0.735^{\star}$ \\
\hline
\end{tabular}

and 14-day self-quarantine period; At the end of the self-quarantine period, the nurse will be tested for COVID-19 infection, and then they are allowed to go home if the result is negative.

\subsubsection{Fear of Being Infected}

Ranked second among nurses' stressors, it belongs to the dimension of conceptual stress. It is related to the high infectiousness of COVID-19 and the current limitations of understanding of the disease. Since the outbreak of COVID-19, the number of medical staff infected has reached 3387, and many have unfortunately passed away. It is highly risky to nurse patients with COVID-19, which requires direct contact and long exposure with the source of infection. Even if the nurses have done a good job of safety protection according to the protocol, due to the complicated nursing process, they are worried that something is not implemented in place, leading to the occurrence of infection, which has caused great pressure on nurses [16]. This requires hospital leaders and nursing managers to strengthen the safety protection of medical staff to ensure "zero infection", and achieve the following five points: 1) Targeted training. Compared with ordinary wards, protection and disinfection Isolation of knowledge and training of high-risk operations under three levels of protection is the top priority [17]. The combination of online theoretical learning, offline simulation training, online theoretical assessment, and one-to-one operation clearance before starting the job is adopted. Cloud classroom, video teaching, scenario simulation and other forms of centralized training, on-site learning, and one-to-one guidance are combined to ensure the learning effect, and only after passing the assessment, they can take the job. 2) Guarantee the supply of protective materials. Establish a ledger for protective products and disinfection products, dynamically grasp the material reserves in the COVID-19 ward, and ensure the basic dosage of protective equipment and the occupational safety of medical personnel. 3) Establish a reasonable physical barrier. It is important to ensure that the ward has a reasonable "clean area, potentially contaminated area, and contaminated area" partition, and have medical staff passages and patient passages to ensure that there is no cross infection. 4) Establish a standardized behavior 
barrier. The behavior of nurses in areas with different cleanliness should follow the corresponding systems and operating procedures to ensure the implementation of various details [18]. 5) Each ward is equipped with full-time personnel for hospital infection control to promote the implementation of the daily prevention and control concept. It is recommended to supervise via surveillance on implementation of disinfection and isolation in the ward, and the standardized process of putting on and taking off of protective equipment. Strengthen the full-process tracking management of medical waste.

\subsubsection{Want to Return and Dare Not/Cannot Go Home}

Ranked third among nurses' stressors, it belongs to the dimension of conceptual stress. In order to ensure safety, the hospital centrally arranged the lives of the medical staff. The nurses wanted to go home to visit their family member, but was worried that it would affect the family. Some communities fear that nurses will bring the virus back to the community and thus restricted the nurses from entering the community. As a result, nurses cannot go home [19]. These all add pressure on the nurses. This requires hospitals and nursing managers to pay attention to: 1) To implement nurses' rotation system: to ensure the physical and mental rehabilitation of nursing staff, implement echelon work system, fixed time, fixed personnel, regular rotation rest, and ensure the rest period of nurses and their family members reunion time [20]. 2) It is necessary to rationally allocate medical resources to ensure that the nurses are properly fed and dressed, and try to solve their family difficulties so that they have no worries. 3) Propagate the knowledge of COVID-19 scientifically, impartially and objectively to the public, so that the public can treat nurses rationally.

\subsubsection{Performing High-Risk Operations (Nebulization, Oral Care, Tracheal Intubation, Etc.)}

Increases the chance of infection: it ranked fourth among nurses' stressors and belongs to the dimension of occupational hazards. These high-risk operations may be splashed by body fluids, blood, and secretions of patients with COVID-19, and aerosols may be generated, which may lead to direct exposure of nurses and increase the risk of infection [21]. This requires hospitals and nursing managers: 1) Define high-risk operations and formulate protection details for high-risk operations. 2) Conduct protective simulation training and occupational exposure emergency drills for high-risk operations, and work normally only after mastering them. 3) Principle of selection of support staff. According to the number and severity of patients, and the operations needed, arrange the corresponding resource to ensure the correct implementation. 4) Ward supervisors should incorporate high-risk operation protection details into the supervision content, and continuously improve to ensure that the prevention and control details of each operation.

\subsubsection{The Stress of Working Environment}

It ranked fifth in the stressor dimension of nurses. Irregular working hours 
(such as unable to eat, drink, go to the toilet, etc.), protective clothing and uncomfortable supplies rank sixth and seventh in the stressor category of nurses. This is related to the long working hours, the need for strict protection, the inability to eat, drink, or go to the toilet during work, and solve the "three emergency" of life. It is also related to the use of protective equipment that causes nasal and facial pressure sores, protective equipment allergies and inability to wear protective equipment correctly cause ischemia and hypoxia [22]. This requires hospitals and nursing managers to take the following actions. 1) Reasonably arrange nursing human resources and working hours: rationally allocate and deploy nurse human resources, and scientifically arrange working shifts and times. The working time of each shift is 4 - 6 hours [23]; Implement mandatory rotation rest; ensure nurses' diet and rest, and implement more humane management. 2) Formulate procedures for the prevention and treatment of head and face skin damage caused by protective equipment, and supervise the implementation to avoid or reduce damage. 3) Establishing health records of nursing staff: The hospital shall set up a psychological assistance team to conduct psychological assessment and guidance for front-line nursing staff. The head nurse shall assess the physical, psychological, family, and menstrual conditions of the nurses daily According to the actual conditions of the nurses and the number of patients, the degree of severity and the difficulty of nursing, the nursing staff should be arranged and dynamically deployed to ensure the safety of the nursing staff [24].

\subsection{Comparison of General Data on Work Stressors and Psychological Behaviors of Nurses in the COVID-19 Ward}

Regarding age, years of service, professional title, educational background, and departments, there is no difference in nurse stressors. While the average score of nurses' stressors is statistically significantly different with different marital status $(\mathrm{P}<0.05)$. Married nurses are more stressed, with stronger psychological and behavioral responses. This may be related to nurses assuming multiple social roles after marriage. They are mothers or fathers, daughters or sons, and they play the main role in the family. When facing the epidemic, nurses on the front line not only have to face the threat of infection alone, but also need to be separated from their families for a long time for the safety of their families, unable to meet with their families, and unable to take care of the elderly and children. Therefore, after marriage, nurses have more concerns, a stronger sense of family responsibility, heavier psychological reactions, and greater pressure [25]. This requires hospital leaders to avoid arranging for both husband and wife to go to the front line support at the same time, to stabilize the family. If it is necessary for both husband and wife to be on the front line at the same time, the hospital's logistics support department should take good care of the elderly and arrange the life and study of their children so that nursing staff have no worries. The rotation system can be implemented so that they can go to work regularly, take regular breaks, and have the opportunity to reunite with their families regularly 
to reduce mental stress. It is necessary to focus on the psychological behavior of nurses after marriage, and provide personalized guidance and intervention to nurses who have psychological behavior deviations.

\subsection{The Self-Evaluation of Psychological Behaviors of Nurses in the COVID-19 Ward Facing Sudden Public Health Emergency and Their Correlation with Nurse Pressure}

The average score of self-evaluation of psychological behaviors of nurses in COVID-19 ward facing emergency public health events is $(1.40 \pm 0.54)$ points, which is a moderate response. The highest dimension of psychological behavior is the fear factor, and the lowest dimension is the compulsive-anxiety factor. The stressor score of nurses working in COVID-19 ward was positively correlated with the psychological behavior score $(\mathrm{P}<0.01)$, that is, the higher the psychological behavior score, the highest stressor score for nurses. This result may be related to the following reasons: 1) Although our hospital is in the epidemic area, the nurses who support the novel corona virus pneumonia disease area have zero infections. The nurses fully realize that as long as standardized protection is required, infection can be avoided. 2) Due to the strong contagiousness of novel corona virus pneumonia, the current limitations of understanding of the disease, and the high-risk operation of nursing for COVID-19 patient, these all increase the uncertainty of infection and cause the fear of nursing staff. 3) Under the current situation of a large number of medical staff infections in Hubei Province, the first-line nurses will also experience psychological changes such as sensitivity, vulnerability, fear, and worry, and even individual nurses will have psychological behavior deviations, leading to bad psychological behaviors. This will directly result in the increase in psychological stress of the nursing staff. In the face of this situation, the manager's work recommendations are as follows: 1) Establish a nurse's psychological file: evaluate the psychological behavior of each nurse and use it as one of the basis for staffing. 2) Establish a psychological behavior control model to conduct personalized psychological counseling: establish a control chart of fear, depression, neurasthenia, compulsive-anxiety, hypochondriasis, and psychological behavior total scores. For nurses with psychological behavior deviations, one-to-one conduct Psychological counseling and intervention. 3) The novel corona virus pneumonia ward should be equipped with fixed psychological staff, regularly carry out mental health assessment, and conduct online and offline psychological consultation and psychological treatment, so as to reduce the psychological and behavioral response of nurses in the COVID-19 ward, thereby ultimately reducing the pressure on the nurses.

\section{Conclusion}

Preventing and controlling the COVID-19 epidemic are a serious challenge for nurses. Faced with the high contagiousness of COVID-19 and the limited knowledge of the disease, nursing staff experience various levels of pressure and emotional stress, which affects their physical and mental health. Therefore, hospital 
administrators should pay attention to the mental health of nurses in the COVID-19 ward, especially nurses with mental behavior deviations and married ones. Psychological counseling and crisis psychological intervention shall be conducted as soon as possible to promote the mental health of the clinical nurses. Due to the constraints of time, investigation area and other objective conditions, this study only investigated clinical nurses in a tertiary hospital in a prefecture-level city in Hubei Province. It is inevitable that there are certain limitations. We hope that more comprehensive research will be carried out in multiple hospitals in different regions.

\section{Conflicts of Interest}

The authors declare no conflicts of interest regarding the publication of this paper.

\section{References}

[1] Khamisa, N., Oldenburg, B., Peltzer, K. and Ilic, D. (2015) Work Related Stress, Burnout, Job Satisfaction and General Health of Nurses. International Journal of Environmental Research and Public Health, 12, 652-666. https://doi.org/10.3390/ijerph120100652

[2] Shahrour, G. and Dardas, L.A. (2020) Acute Stress Disorder, Coping Self-Efficacy and Subsequent Psychological Distress among Nurses amid Covid-19. Journal of Nursing Management, 28, 1686-1695. https://doi.org/10.1111/jonm.13124

[3] General Office of the State Health and Health Commission (2020) Notice Issued by the General Office of the State Health and Health Commission on Further Strengthening the Protection of Medical Personnel during the Period of Epidemic Prevention and Control.

[4] Ma, D.F., Wang, Y.T., Zhang, Q.W., Chang, X.Y. and Zhu, Y.J. (2019) Status Quo and Influencing Factors of Scientific Research Stressors for 275 Operating Room Nurses in Tertiary Hospitals. Journal of Nursing Science, 26, 65-71.

[5] Jing, X.B., Liu, H., Fang, X.J., Gao, T.T., Li, C.P. and Fan, X.T. (2016) A Study on the Correlation between Work Stressors Psychological Capital and Career of Nurses in Critical Care Specialty. Nursing Research: Late Edition, 30, 3348-3351.

[6] Sun, X.F., Ren, Y.B. and Luan, X.R. (2015) Investigation on Job Stress Sources and Job Burnout of Nurses with Different Educational Backgrounds. Chinese Journal of Nursing Management, 15, 1000-1003.

[7] Ezenwaji, I.O., Eseadi, C., Okide, C.C., Nwosu, N.C., Ugwoke, S.C., Ololo, K.O., et al. (2019) Work-Related Stress, Burnout, and Related Sociodemographic Factors among Nurses: Implications for Administrators, Research, and Policy. Medicine, 98, e13889. https://doi.org/10.1097/MD.0000000000013889

[8] Zhang, S.P. (2017) The Correlation between Nurses' Psychological Tolerance and Work Stressors. Nursing Research, 31, 1124-1126.

[9] Chen, Q., Gu, L.H. and Shen, M.F. (2019) The Correlation between Job Burnout and Stressors of Community Nurses in Changshu City, Jiangsu Province. Journal of Nurse Advancement, 34, 93-97.

[10] Feng, P., Zhang, W.Y., Zhang, L., Sun, Y., Qiao, S. and Zhang, L.J. (2016) Investigation of Nurses' Stressors during the Treatment of a Group of Patients with Severe Burns. Journal of Nursing Management, 16, 174-175. 
[11] Liu, C. (2018) A Qualitative Study on Stress Sources and Coping Styles of Rotating Nurses in Intensive Care Unit. Journal of Nurse Advancement, 33, 2194-2196.

[12] Gao, Y., Xu, M.Z., Yang, Y.F. and Yao, K.N. (2004) Study on Coping Styles and Related Factors of College Students during SARS. Chinese Journal of Medical Ethics, 17, 60-63.

[13] Zhang, Z.J. (2005) Behavioural Medicine Questionnaire. Chinese Medical Electronic Audio and Video Press, Beijing.

[14] Turale, S., Meechamnan, C. and Kunaviktikul, W. (2020) Challenging Times: Ethics, Nursing and the Covid Pandemic. International Nursing Review, 67, 164-167. https://doi.org/10.1111/inr.12598

[15] Cai, H., Tu, B., Ma, J., Chen, L., Fu, L., Jiang, Y. and Zhuang, Q. (2020) Psychological Impact and Coping Strategies of Frontline Medical Staff in Hunan between January and March 2020 during the Outbreak of Coronavirus Disease 2019 (COVID-19) in Hubei, China. Medical Science Monitor, 26, e924171. https://doi.org/10.12659/MSM.924171

[16] Stelnicki, A.M., Carleton, R.N. and Reichert, C. (2020) Nurses' Mental Health and Well-Being: Covid-19 Impacts. The Canadian Journal of Nursing Research, 52, 237-239. https://doi.org/10.1177/0844562120931623

[17] Guidelines for Nursing Work in West China Hospital/West China Nursing College (2020) COVID-19 Prevention and Control Hospital Nursing Work Guide. Version 1, Sichuan Science and Technology Press, Chengdu.

[18] Respiratory Critical Care Medicine Group of Respiratory Society of Chinese Medical Association and Critical Care Medicine Working Committee of Respiratory Physicians Branch of Chinese Medical Doctor Association (2019) Consensus of Experts on the Clinical Specification and Application of Transnasal High Flow Humidification Oxygen Therapy for Adults. Chinese Journal of Tuberculosis and Respiratory Diseases, 42, 83-91.

[19] Yang, X., Li, S.J., Hua, L. and Zhang, Y. (2003) Organization and Management of SARS Ward in General Hospital. Chinese Journal of Nursing, 38, 545-546.

[20] Ross, J. (2020) The Exacerbation of Burnout during Covid19: A Major Concern for Nurse Safety. Journal of PeriAnesthesia Nursing, 35, 439-440. https://doi.org/10.1016/j.jopan.2020.04.001

[21] General Office of the State Health and Health Commission (2020) Circular of the General Office of the National Health and Health Commission on the Publication of the Programme for the Diagnosis and Treatment of Pneumonia with New Coronary Virus Infection Trial. 7th Edition, Ministry of Health and Health, Beijing.

[22] Kuo, F.L., Yang, P.H., Hsu, H.T., Su, C.Y., Chen, C.H., Yeh, I.J., et al. (2020) Survey on Perceived Work Stress and Its Influencing Factors among Hospital Staff during the Covid-19 Pandemic in Taiwan. The Kaohsiung Journal of Medical Sciences, 36, 944-952. https://doi.org/10.1002/kjm2.12294

[23] Zhang, J.Y., Yan, R.G. and Zhang, Y. (2004) Organization and Management of the Establishment of SARS Ward in Sanatorium. Chinese Journal of Convalescent Medicine, 13, 40-41.

[24] Luo, C. (2020) Management of a Nursing Unit in a Temporary COVID-19 Specialized Hospital in Wuhan, China. Disaster Med. Public Health Prep. 12, 1-7. https://doi.org/10.1017/dmp.2020.373

[25] Labrague, L.J. and Santos, J. (2020) Prevalence and Predictors of Coronaphobia among Frontline Hospital and Public Health Nurses. Public Health Nursing, 23, 1-8. https://doi.org/10.1111/phn.12841 